\title{
Autonomous System Network Topology Discovery Algorithm Based On OSPF Protocol
}

\author{
Xingchao $\mathrm{Ma}^{1, \mathrm{a}^{*}}$, Geming $\mathrm{Xia}^{2, \mathrm{~b}}$ \\ ${ }^{1,2}$ College of Computer, National University of Defense Technology, Changsha, China

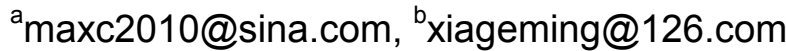

\begin{abstract}
Keywords: Network topology discovery, OSPF protocol, Autonomous system, LSA.
Abstract. The existing algorithms of network topology discovery are mainly based on Internet control message protocol (ICMP) and simple network management protocol (SNMP). These algorithms are active network topology discovery methods which can inject new traffic into the Internet and affect the network performance. In the paper we present an algorithm of network topology discovery based on open shortest path first (OSPF) protocol. As one kind of active network topology discovery methods, the algorithm of network topology discovery based on OSPF protocol doesn't inject new traffic into the network. Moreover, this algorithm of network topology discovery based on OSPF protocol can make up the deficiencies of existing techniques of network topology discovery. We suspect that this algorithm of network topology discovery based on OSPF protocol will be widely used.
\end{abstract}

\section{Introduction}

Network topology discovery is a technology that automatically finds the computer network entities (routers, hosts, subnet, etc.) and the connection between entities without manual intervention. Network topology discovery is the basic function of network management tools today. This technology plays an important role in optimizing the network configuration, finding the bottleneck of the network, locating the network failure and finding the potential risks that may exist in the network.

The traditional network topology discovery methods are mainly based on ICMP protocol and SNMP protocol $[1,2]$. In recent years, the network topology discovery technology has been widely studied in the domestic and international academic circles [3, 4].

\section{Related Work}

\section{Analysis of Network Topology Discovery Technology}

Network topology discovery is one of the core technologies in the field of computer network management. And it has been studied for many years both at home and abroad. Different classifications are listed below.

Physical Network Topology Discovery and Logical Network Topology Discovery

Physical network topology is a physical connection between real network devices. For example, the connection between the switch and the switch, the switch and the host. Logical network topology uses the IP address of the network equipment to find the logical connection relationship between network devices, including connections between router and router, router and subnet. Physical topology discovery technology works on the data link layer, while logical network topology discovery on the IP layer. In general, physical network topology discovery can be realized in local area network (LAN). And logical network topology is realized in the wide area network.

\section{Active and Passive Network Topology Discovery Methods}

Active network topology discovery method observes and records the results by sending monitor data packets to the target network. By using this method the behaviors of the network users and structure of the network can be studied. Active network has its shortage. When sending monitor data packets in the network, extra network load increases. The accuracy of the topology discovery is not good because of the increasing network load. However, passive network topology discovery method has no 
effect on the network topology discovery. In this method, monitors which are set up in the target network record the data of the network and extract information relate to topology. With this information the topology of the network can be restored. The passive network topology discovery method doesn't increase network load, having no effect on the performance of the network.

Topology Discovery Base on SNMP and ICMP Protocol

In recent year topology discovery technologies base on SNMP and ICPM protocol are the most widely used. At the same time they are the most widely studied both at home and abroad.

\section{Analysis of Topology Discovery Technology Based on OSPF Protocol}

Routers rely on routing protocols to generate their own routing table. Routing protocols maintain the normal order of the network. If we can get the data of routing protocols, we can learned how routers connect to each other and their respective subnet, and the topology of the network at the same time. There are two different dynamic routing protocols: Distance Vector Protocol and Link State Protocol.

Distance Vector Protocol (DVP). Routers running a DVP transfer some or all of the routing tables directly to their neighboring routers. If we can obtain the data of the DVP, we can obtain the routing table, then with the routing table we can restore the topological structure. DVP are very familiar with network topology discovery technology base on SNMP protocol.

Link State Protocol (LSP). The LSP transfers the information of the network topology, which is also called the link state information. Routers learn the topology of the entire network, and then calculate the routing table based on the topology information.

OSPF protocol is a typical LSP. Network topology discovery based on OSPF learns the connections between the topological nodes by analyzing the data of OSPF protocol [5].

Link-State Algorithm. In OSPF protocol, routers use link state advertisements (LSA) to describe the link state information. If we can obtain the LSA, we can get the information of the network topology. LSAs are exchanged by flooding in autonomous system (AS). Flooding means that a router generates LSA packets and transfers them to all its neighboring OSPF routers. Neighboring OSPF routers update their link state database (LSD) according to the received LSA packets. After that these neighboring OSPF routers transfer these LSA packets to their neighboring routers. This process continues till the LSA packets are transferred to all the OSPF routers and the network go into a stable statement. Then all the OSPF routers calculate their routing table according to their LSD.

In order to simplify routing table and improve the operation speed of router, OSPF protocol divides an AS into many areas. Each area is connected by the area border router (ABR). LSAs only floods in the one area. This flooding method makes sure that every router can obtain all the LSAs from other routers in the same area. In other words, every LSA will go through the link between any two routers in the same area. Thus, only one probe is needed to set up and then the whole LSAs will be grabbed in an area.

Classifications of OSPF Protocol Packet. There are five different types of OSPF protocol packets. Firstly, the hello packets. They are used to build up connections to their neighbor nodes. Secondly, the database description (DBD) packets. They are used to test the synchronization of the link state database. Thirdly, the link state request (LSR) packets. Fourthly, the link state update (LSU) packets. Fifthly, the link state acknowledgement (LSAck) packets.

LSU packets. The fourth type of OSPF protocol packet, LSU, plays an important role in the OSPF protocol because all the LSAs are encapsulated in it. The format of LSU packet is shown in Fig.1. 


\begin{tabular}{|l|c|c|}
\multicolumn{1}{|c|}{7} & 15 \\
\hline Version & 4 & Packet length \\
\hline \multicolumn{3}{|c|}{ Router ID } \\
\hline \multicolumn{3}{|c|}{ Area ID } \\
\hline Checksum & $\begin{array}{c}\text { Authentication } \\
\text { type }\end{array}$ \\
\hline \multicolumn{3}{|c|}{ Authentication } \\
\hline \multicolumn{3}{|c|}{ Number of LSAs } \\
\hline LSA \\
\hline LSA \\
\hline LSA \\
\hline
\end{tabular}

Fig.1 The format of LSU packet

Fig. 1 shows that all OSPF packets have a 24 byte header, and every LSU packet can carry more than one LSA.

Each LSA has a 20 byte header. And the load size of LSA varies due to different LSA type. At present, OSPF has defined 11 kinds of LSAs, but only two types of them can be used in topology discovery. There are router LSA and network LSA.

Router LSA. Every router generates router LSA. Router LSA is the most important LSA in the network topology discovery. What's more, router LSA only transfers in one area and doesn't go through the ABR. when it comes to the ABR, ABR works for more than one area. ABR will generate one LSA per area. Specially, this LSA describes the information between the ABR and the corresponding area, but not the whole information between the ABR and all its linking areas. The format of router LSA is shown in Fig.2.

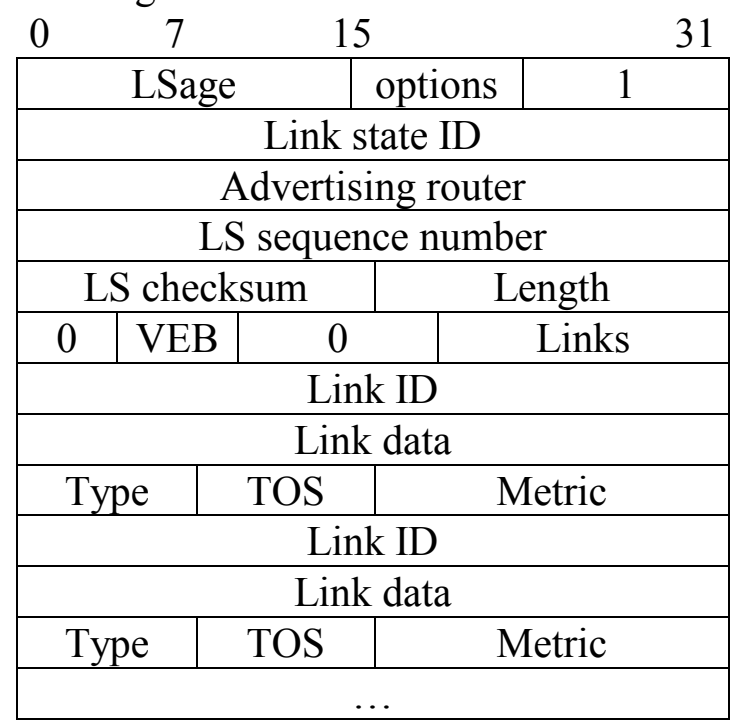

Fig. 2 The format of router LSA

Router LSA describes a link with five elements. They are type, link ID, link data, metric and type of service (TOS). One router LSA can include couples of arrays with these five elements but no more than the number of the link the router has.

We can get two nodes and one link by analyzing the router LSA header and the array with the five elements. One node comes from the advertising router field of the router LSA header. When type field equals to 1, link ID must be a router which connect to the advertising router. When type field equals to 2 , one transit connecting to the advertising router can be discovered. And this transit includes link ID and link data. When type field equals to 3, a subnet known by link ID and link data can be discovered. And this subnet connects to the advertising router. When type field equals to 4 , link ID can be discovered as a router which connects to the advertising router by a virtual link. 
Network LSA. Network LSA is generated by the designed router (DR) in the transit network. It is used to describe the routing information of the transit. Also it transfers in one area and doesn't go through the ABR. There are three important fields in the network LSA. They are advertising router, network mask and attached router. Network mask is the mask of the transit. Attached routers are the router IDs of the routers forming the transit network. The format of network LSA is shown in Fig.3.

\begin{tabular}{|c|c|c|}
\hline 7 & & \\
\hline LSage & options & 2 \\
\hline \multicolumn{3}{|c|}{ Link state ID } \\
\hline \multicolumn{3}{|c|}{ Advertising router } \\
\hline \multicolumn{3}{|c|}{ LS sequence number } \\
\hline LS checksum & & \\
\hline \multicolumn{3}{|c|}{ Network mask } \\
\hline \multicolumn{3}{|c|}{ Attached router } \\
\hline
\end{tabular}

Fig.3 The format of network LSA

By analyzing the network LSA, we can discover one node, the advertising router. Also we can discover the attached router from the transit network and the network mask field of the transit network.

\section{Algorithm Design}

Protocol data acquisition. Protocol data acquisition is the basis of the network discovery technology based on OSPF protocol. Before we obtain the protocol data, we learn how the basic algorithm of OSPF protocol works.

LSA Update. The OSPF protocol provides a lot of LSA updates. Here are three common updates.

1. Router initialization update. When a router is newly added to the network, the router will generate a LSA.

2. Triggered update. When the network state changes (such as increasing or reducing routers and link state change), routers will detect the changes and send triggered updates. This triggered update ensure the real-time characteristic of the network topology discovery.

3. Periodic updates. A LSA has its living time (link state refresh time, 30mins). When living time is up, routers will send periodic updates. Thus, we can obtain all the LSAs in the target network in 30 minutes. Periodic updates ensure the integrity of the network topology discovery.

The main flow of network topology discovery algorithm based on OSPF protocol is shown in Fig.4. In this algorithm, router LSA and network LSA are collected and analyzed. Different actions are taken due to the kind of the LSA. When all the LSAs are analyzed, the algorithm ends. Fig.4 is the main flow of this algorithm. 


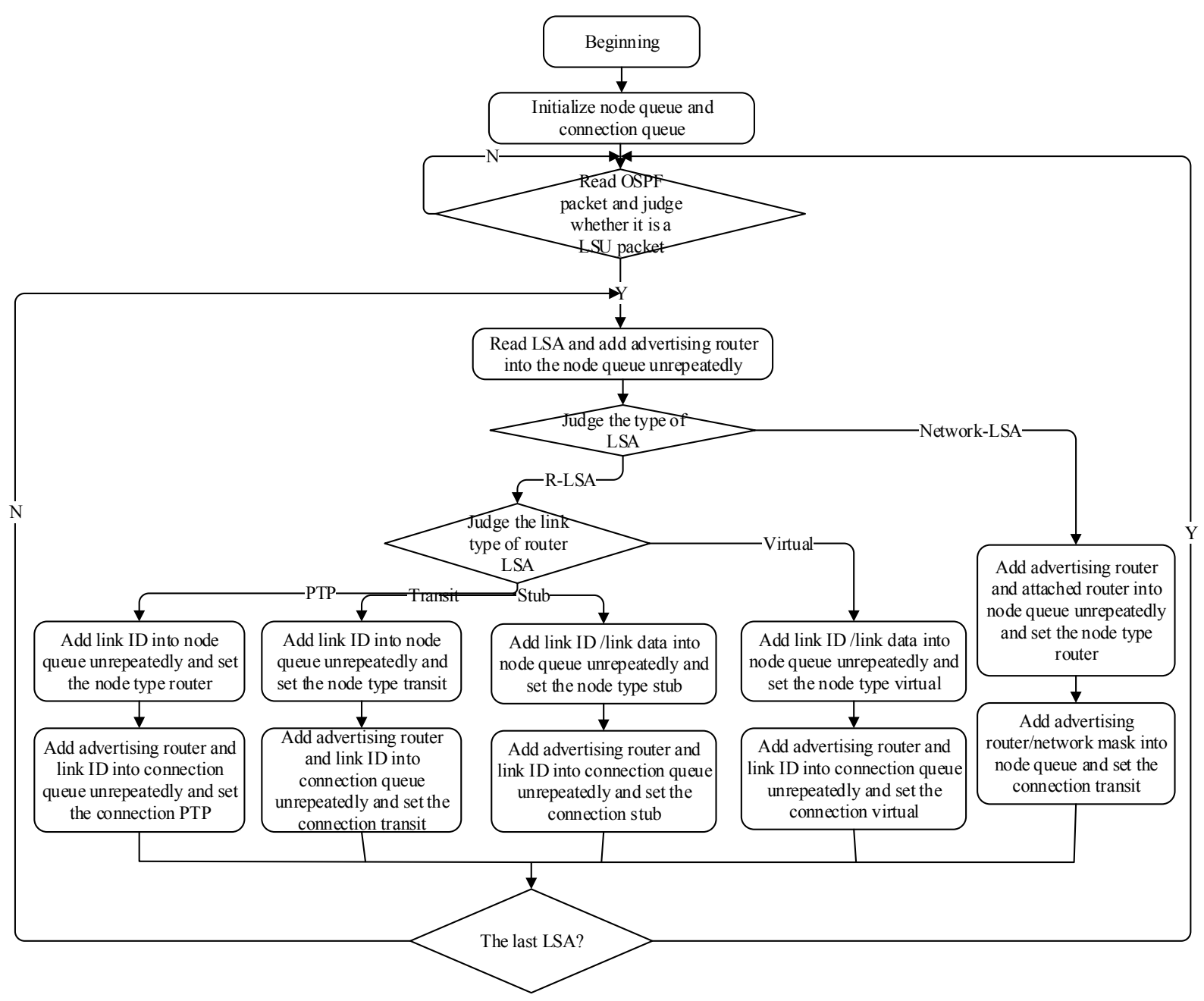

Fig.4 The main flow of network topology discovery based On OSPF protocol

\section{Experiment}

Implement. We have realized the network topology discovery technology based on OSPF protocol. This technology has achieved good results in the practical engineering. The function of the network topology discovery system can be divided into three parts. More details of the system are shown in Fig.5. 


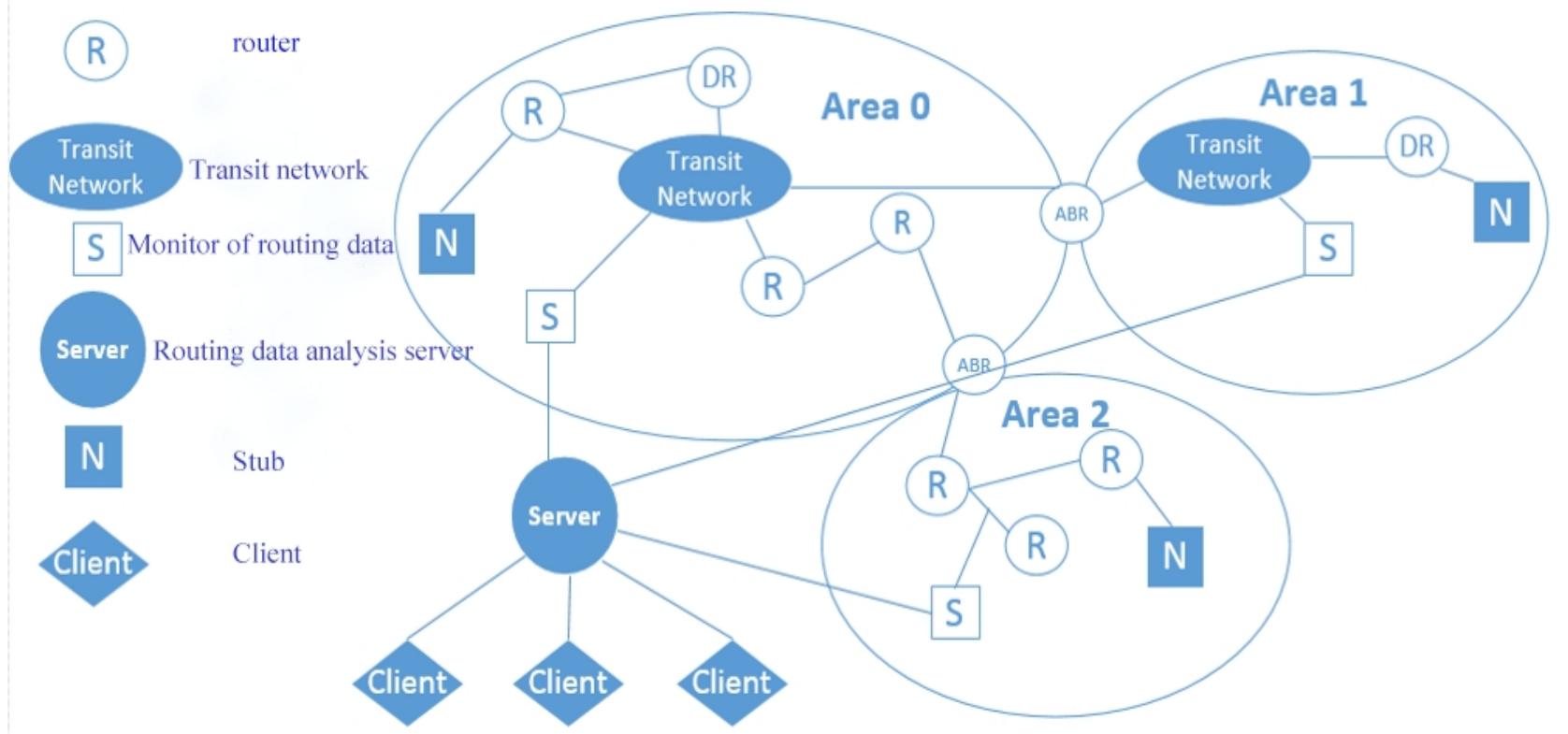

Fig.5 The topology discovery system

Monitor of routing data. Every OSPF area in the target network deploys one monitor of routing data. This monitor can record the LSU packets of the OSPF protocol and send them to the routing data analysis server. Then the server will analyze the data.

Routing data analysis server. Routing data analysis server collects and analyzes the OSPF packets sent from the monitors, and then the server records the protocol field into the database. In the database, two tables are created. One is for router LSA, and one for network LSA. Both of the tables have a link state age (LSage) field. When receiving the LSA updates, the server will check whether the LSA updates are the same with the records in the database. No operation if they are the same. If they are not the same, the server will record the LSA updates into the database and notice the topology discovery clients that new link information are found.

Topology discovery client. The main flow of the algorithm in Fig.4 is carried out in the client. The topology discovery clients read the topological data from the database in the server and analyze it to obtain the network nodes and their connections. And then the clients automatically draw a network topology. The client keep in touch with the server. When receiving LSA updates, the server will notice the clients and the clients will read the updates. This method can ensure the real-time characteristic of the topology discovery. Fig.6 is the result of the network topology discovery. 


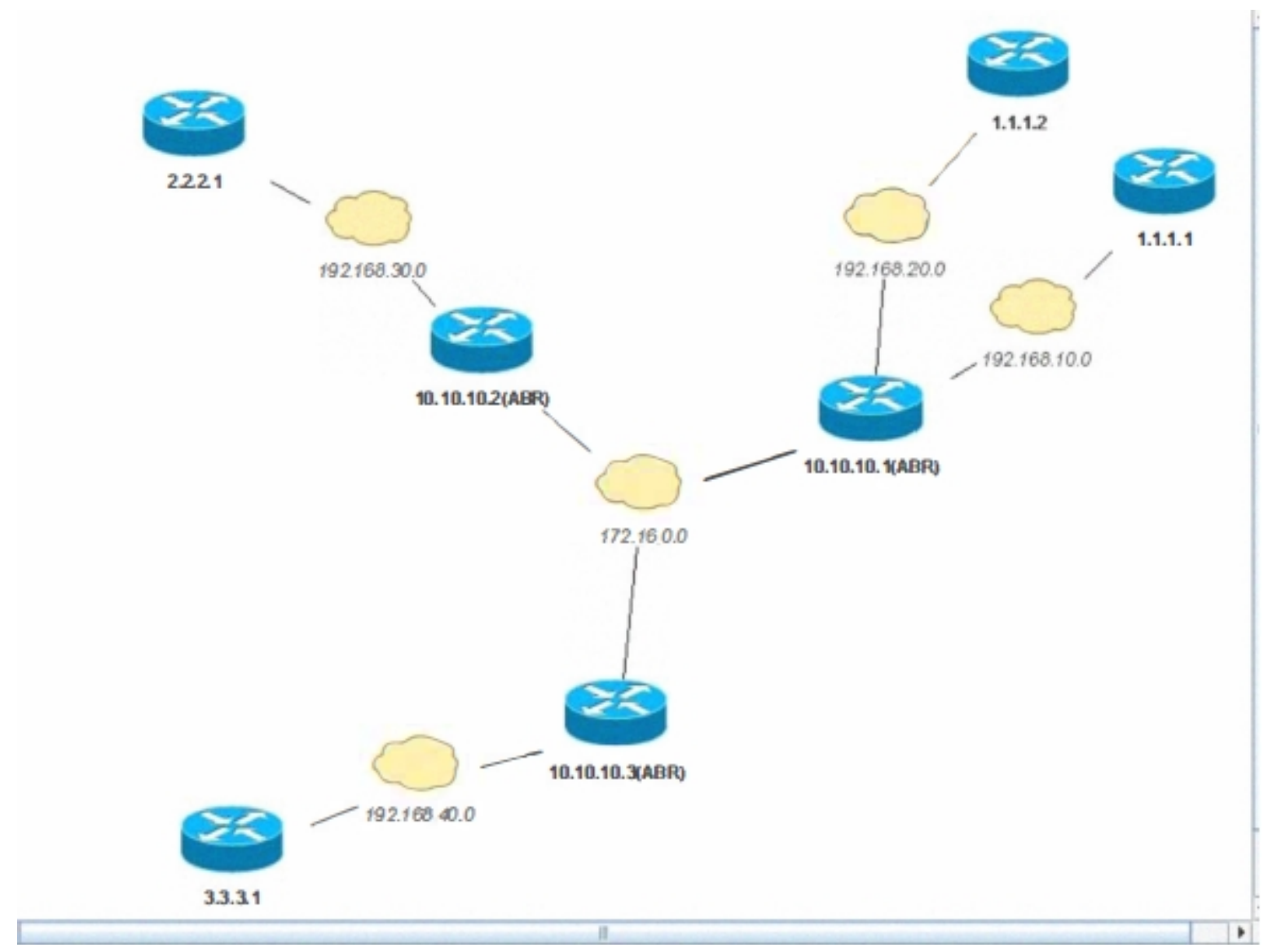

Fig.6 The result of the network topology discovery

\section{Conclusion}

In this paper we show the network topology discovery technology based on OSPF. This technology gets the topology information through analyzing Router-LSAs and Network-LSAs which are carried by LSU packet in OSPF protocol to realize network topology. Network topology discovery based on OSPF protocol uses passive monitoring method to obtain the data. This discovery technology has the characteristics of low load. What's more, OSPF protocol itself can ensure the accuracy, integrity and real-time of topology discovery. The OSPF protocol has become one of the largest and most widely used interior gateway protocol (IGP) routing protocols which are used in wide area network and the enterprise network in the Internet. Network topology discovery technology based on OSPF protocol has broad application prospect.

In the future, we will study the network topology discovery technology based on the comprehensive analysis of multiple routing protocols, especially for the comprehensive analysis of the OSPF, border gateway protocol (BGP) to adapt to more large-scale network topology discovery.

\section{References}

[1] J. van der Geer, J.A.J. Hanraads, R.A. Lupton, The art of writing a scientific article, J. Sci. Commun. 163 (2000) 51-59.

[1] YANG An-yi, ZHU Hua-qing, WANG Ji-Iong. Improved SNMP-based network topology discovery algorithm and its implementation [J]. Computer Applications, 2009, 27(10): 2412-2419.

[2] SHI Yong-ge, CHEN Xiao-fan. Analyses and improvement of topological discovery algorithm based on network [J]. Computer Engineering and Design, 2007, 28(12): 2848-2850.

[3] WANG Hui, LUO Jun-yong, KOU Xiao-rui. Network Topology Algorithm Based on OSPF Protocol Packet [J]. Computer Engineering, 2008, 34(6): 103-105. 
[4] NI Yong, SHI Huai-zhou, ZHU Pei-dong. Construct Network Topology Based on OSPF Database [J]. Computer Technology and Development, 2009, 19(3): 1-4.

[5] Moy J. OSPF Version 2, RFC 2328[S]. The Internet Society: Network Working Group, 1998. 